\title{
Semiclassical spin damping: Superradiance revisited
}

\author{
Petr A. Braun \\ Department of Theoretical Physics, Institute of Physics, Saint-Petersburg University, Saint-Petersburg, 198904 Russia \\ Daniel Braun, Fritz Haake, Joachim Weber \\ Fachbereich Physik, Universität-Gesamthochschule Essen, 45117 Essen, Germany
}

\begin{abstract}
A well known description of superradiance from pointlike collections of many atoms involves the dissipative motion of a large spin. The pertinent "superradiance master equation" allows for a formally exact solution which we subject to a semiclassical evaluation. The clue is a saddle-point approximation for an inverse Laplace transform. All previous approximate treatments, disparate as they may appear, are encompassed in our systematic formulation. A byproduct is a hitherto unknown rigorous relation between coherences and probabilities. Our results allow for generalizations to spin dynamics with chaos in the classical limit.
\end{abstract}

PACS numbers: 42.50F, 03.65.Sq 


\section{INTRODUCTION}

Dissipative motion of large spins was first seen in experiments on superradiance or superfluorescence (For extensive reviews see Refs. [1,2]), after being proposed a lot earlier by Dicke [3]. The so called superradiance master equation proposed in 4.5. has since become a standard tool for describing the collective dynamics of identical superradiating atoms in the small-sample limit. Formally speaking, it provides a quantum treatment of a large spin with conserved square, $\mathbf{J}^{2}=j(j+1)$, with the quantum number $j$ capable of taking on positive half integer or integer values up to half the number of atoms $N$. The origin of such an angular momentum lies in the familiar formal equivalence of a single two-level atom to a spin- $\frac{1}{2}$. In (semi)classical parlance, the spin in question is called the Bloch vector whose $z$-component measures the energy stored in atomic excitation while the transverse components are related to the dipole element responsible for the atomic transition. More or less everything worth knowing about the superradiance master equation in relation to the numerous superfluorescence experiments has been worked out more than a decade ago.

When we pick up the thread now our motivation is not to better explain anything previously observed, but rather the expectation of new experiments involving dissipative motion of large spins constituted by many identical two-level atoms, albeit motions that would have a chaotic classical limit and display quantum manifestations of chaos when the spin quantum number $j$ is of the order of several hundreds or thousands. When beginning to look into such dynamics [6] we found, somewhat to our surprise, that previous treatments of the superradiance master equation were so directly geared to the specifics of superradiant pulses as transient events that new questions do indeed require some new theoretical work. In particular, the semiclassical limit of large $j$ deserves systematic attention and turns out to harbor one or the other surprise which we begin to uncover in the present paper.

The large- $j$ limit can be approached through the rigorous solution of the master equation which was known from the very beginning [4, and we shall actually follow that path here. Strangely enough, up to now that rigorous solution has mostly been looked upon as a curiosity rather than a useful starting point of analytic work; even numerical evaluations were disfavored against routines for solving coupled differential equations for density matrix elements in some representation.

We propose to show that the large- $j$ limit is very conveniently accessed by subjecting the rigorous Laplace transformed density matrix to a saddle-point evaluation of the inverse Laplace transformation. More specifically, we carry out this program in the eigenrepresentation of $J_{z}$ and $\mathbf{J}^{2}$ for the density matrix $\left\langle j m|\rho(t)| j m^{\prime}\right\rangle$ and the propagator relating that density matrix to its initial form $\left\langle j m|\rho(0)| j m^{\prime}\right\rangle$. The saddle-point result turns out reliable provided that not only $j$ is large but also the difference between the initial and final eigenvalues of $J_{z}$, i.e. $\left|m-m^{\prime}\right| \gg 1$. That restriction unfortunately affects the propagator at early times while most of the probability still resides in levels $m$ close to the initial $\mathrm{m}^{\prime}$. We therefore establish an independent early-time propagator, show its agreement with the saddle-point version in a certain time span and finally combine the two to an explicit expression of uniform validity.

Our uniform propagator turns out to systematically encompass previous asymptotic results. Among these is, trivially, the fully classical behavior arising in the limit $j \rightarrow \infty$ as long as the initial state is not too close to the state of full initial excitation $m=j$ which in the classical limit is an infinitly long-lived state of marginal equilibrium. The classical behavior in question is that of an overdamped pendulum. The pertinent equation of motion for the so-called Bloch angle $\theta$ (defined through $\cos \theta=\lim _{j \rightarrow \infty}\left\langle J_{z}(\tau)\right\rangle / j$ ) reads, with $\tau$ denoting a suitably scaled time, $\frac{d}{d \tau} \theta=\sin \theta$; the the well known solution is

$$
\tan \frac{\theta(\tau)}{2}=\mathrm{e}^{\tau} \tan \frac{\theta(0)}{2}
$$

Furthermore, we recover the random-jitter picture first suggested in [7, 8 and the ensuing distribution of delay times as well as the scaling results for time dependent expectation values of products of the observables $J_{x}, J_{y}, J_{z}$ obtained by somewhat hit-and-run methods in [9,10].

An interesting byproduct of our investigation is an exact relation between diagonal and offdiagonal elements of the density matrix in the $j m$-basis, which to the best of our knowledge has previously gone unnoticed. One may thus confine all work towards solving the master equation to the probabilities $\langle j m|\rho(t)| j m\rangle$ and eventually obtain the coherences $\left\langle j m|\rho(t)| j m^{\prime}\right\rangle$ through the relation in question.

A subsequent paper will deal with the large- $j$ limit with the WKB method.

\section{MASTER EQUATION AND DISSIPATIVE PROPAGATOR}

The two states of an atom resonantly coupled to a mode of the electromagnetic field may be thought of as the states of a spin- $\frac{1}{2}$, and all observables of the effective two-level atom can be represented as linear combinations of 
unity and the three spin operators $J_{x}, J_{y}, J_{z}$. In particular, the energy may be associated with $J_{z}$ and the other two spin operators with the atomic dipole moment. If $N$ such atoms, all identical, couple collectively to the electric field $E$ one has an interaction Hamiltonian $\propto-J_{x} E$ where $J_{x}=\sum_{\mu=1}^{N} J_{x}^{\mu}$ is the sum of all single-atom contributions; similarly, one has a global atomic energy $\propto J_{z}=\sum_{\mu=1}^{N} J_{z}^{\mu}$. The collective spin operators obey the familiar angularmomentum commutation relations $\left[J_{x}, J_{y}\right]=\mathrm{i} J_{z}$ etc. The Hilbert space for the $N$ atoms is $2^{N}$ dimensional but falls into subspaces not connected by the collective observables $J_{i}$; each subspace has fixed $\mathbf{J}^{2}=j(j+1)$ with nonnegative integer or half-integer $j$ not exceeding $N / 2$. The $(2 j+1)$ states in the $j$ th subspace are conveniently taken as the eigenstates $|j m\rangle$ of $J_{z}$ with eigenvalues $m=-j,-j+1,-j+2, \ldots, j$. The highest energy may be associated with $m=j$ whereupon the ground state has $m=-j$. In particular, the subspace with $j=\frac{N}{2}$ consists of $N+1$ states which are all totally symmetric in all atoms; that space may be singled out experimentally by preparing all atoms in their lower state.

In the superradiance experiments of Ref. [11] a single mode of the electromagnetic field within a resonator was coupled to $N$ two-level atoms such that the dynamics was that of the so-called Jaynes-Cummings model, with dissipation included to account for field losses from the resonator. In the limit of overdamped Rabi oscillations the field mode can be eliminated adiabatically. A master equation for the atomic density operator thus results [ 4 河] of which we shall consider the low-temperature version, thus forbidding the atoms to pick up thermal photons from the environment,

$$
\frac{d}{d t} \hat{\rho}=\kappa\left\{\left[J_{-}, \hat{\rho} J_{+}\right]+\left[J_{-} \hat{\rho}, J_{+}\right]\right\} ;
$$

here $J_{ \pm}=J_{x} \pm i J_{y}$ are the familiar raising and lowering operators and $\kappa$ measures the rate of photon loss from the cavity.

In the basis set $|j m\rangle$ we obtain from (2.1) a set of equations for the elements $\rho_{m_{1} m_{2}}=\left\langle j m_{1}|\hat{\rho}| j m_{2}\right\rangle$ of the density matrix,

$$
\dot{\rho}_{m_{1} m_{2}}=2 \kappa\left[\sqrt{g_{m_{1}+1} g_{m_{2}+1}} \rho_{m_{1}+1, m_{2}+1}-\frac{g_{m_{1}}+g_{m_{2}}}{2} \rho_{m_{1}, m_{2}}\right]
$$

in which $g_{m}$ denotes the "rate function"

$$
g_{m}=j(j+1)-m(m-1) .
$$

The diagonal element $\rho_{m m}$ of the density matrix gives the probability to find the system of atoms in the state $|j m\rangle$; the elements $\rho_{m_{1} m_{2}}$ with $m_{1}-m_{2} \neq 0$ will be referred to as coherences. It is worth noting a certain unidirectionality of the flow of probability and coherence, downwards the $m$-ladder, the physical origin of which is of course the low-temperature limit mentioned above. A further important feature of the system (2.2) is that the density matrix elements with different $m_{1}-m_{2}$ evolve independently. To make that independence manifest it is convenient to introduce the quantum numbers

$$
m=\frac{m_{1}+m_{2}}{2}, \quad k=\frac{m_{1}-m_{2}}{2}
$$

which can be simultaneously either integer or half-integer. Accounting for

$$
\frac{g_{m_{1}}+g_{m_{2}}}{2}=g_{m}-k^{2}
$$

and changing the notation $\rho_{m_{1} m_{2}}$ for the density matrix element to $\rho_{m}^{k}$ we can rewrite the master equation as

$$
\frac{d \rho_{m}^{k}}{d t}=2 \kappa\left[\sqrt{g_{m+k+1} g_{m-k+1}} \rho_{m+1}^{k}-\left(g_{m}-k^{2}\right) \rho_{m}^{k}\right] .
$$

It is now indeed obvious that the "skewness" $k$ enters only as a parameter.

The linear relation between the density matrices at the current time and at the initial moment,

$$
\rho_{m}^{k}(t)=\sum_{n} D_{m n}^{k}(t) \rho_{n}^{k}(0)
$$

defines the $k$-dependent matrix $D_{m n}^{k}(t)$ which will be called the dissipative propagator. Its column corresponding to a certain fixed $n$ can be regarded as the solution of the master equation (2.6) corresponding to the initial condition $\rho_{m}^{k}(0)=\delta_{m n}$. Due to the unidirectionality of the master equation it is obvious that $D_{m n}^{k}=0$ if $m>n$. We shall drop the superscript $k$ in the case $k=0$, i.e. when the diagonal elements of the density matrix are considered. 


\section{SADDLE-POINT ASYMPTOTICS OF THE DISSIPATIVE PROPAGATOR}

There have been a number of successful attempts to treat the large- $j$ limit of the superradiance problem [4,9, 10,7,8,1]. These were concerned with the solution of the master equation for certain particular cases or directly aimed at specific average properties of the process. The purpose of the present paper is to establish uniform asymptotics of the dissipative propagator without such restrictions. We use the exact solution of the master equation in the form of the Laplace integral which was obtained long ago [4] but remained largely unexplored. Previously established results for the propagator, the distribution of delay times, and time dependent expectation values follow from our uniform asymptotic propagator.

Before embarking on our proposed asymptotic adventure it is convenient to adopt the parameter

$$
\sqrt{j(j+1)} \approx j+\frac{1}{2} \equiv J
$$

as a measure of the "size" of the angular momentum; the semiclassical formulae to be established take a prettier form if we use $J$ rather than $j$.

\section{A. Laplace representation of the exact propagator}

Following -4 let us recall the Laplace integral representation of the propagator. Defining the Laplace image as $\mathcal{D}_{m n}^{k}(z)=\int_{0}^{\infty} \mathrm{e}^{-z t} D_{m n}^{k}(t) d t$ we turn our master equation into a recursion relation with the easily found solution

$$
\mathcal{D}_{m n}^{k}(z)=\frac{1}{2 \kappa \sqrt{g_{m-k} g_{m+k}}} \prod_{l=m}^{n} \frac{\sqrt{g_{l-k} g_{l+k}}}{\frac{z}{2 \kappa}+g_{l}-k^{2}} .
$$

To get the dissipative propagator itself we invert the Laplace transform. Introducing a scaled time

$$
\tau=2 \kappa J t
$$

and the quantity

$$
Q_{m n}=\prod_{l=m+1}^{n} g_{l}=\frac{(j+n) !(j-m) !}{(j+m) !(j-n) !}
$$

we bring our propagator to the form

$$
D_{m n}^{k}(\tau)=\frac{\sqrt{Q_{m-k, n-k} Q_{m+k, n+k}}}{2 \pi i} \int_{b-i \infty}^{b+i \infty} d v \mathrm{e}^{\tau v / J} \prod_{l=m}^{n} \frac{1}{v+g_{l}-k^{2}}
$$

where $b$ should be larger than the largest pole in the denominator.

\section{B. Relation between densities and coherences}

An unexpected new result of the representation (3.5) is an identity connecting the propagators for the diagonal and for the off-diagonal elements of the density matrix,

$$
D_{m n}^{k}(\tau)=D_{m n}(\tau) \frac{\sqrt{Q_{m-k, n-k} Q_{m+k, n+k}}}{Q_{m n}} \mathrm{e}^{k^{2} \tau / J}
$$

For the proof it is sufficient to shift the integration variable in (3.5) to $\bar{v}=v-k^{2}$. Alternatively, the connection between the diagonal and off-diagonal density matrix elements can be checked by entering the master equation with the ansatz

$$
\rho_{m}^{k}=\frac{(j+m) !}{(j-m) !} \frac{\sqrt{(j-m-k) !(j-m+k) !}}{\sqrt{(j+m-k) !(j+m+k) !}} \mathrm{e}^{2 \kappa k^{2} t} \tilde{\rho}_{m}(t)
$$


the new unknowns $\tilde{\rho}_{m}(t)$ then turn out to evolve in time like probabilities, i.e. to obey (2.6) for $k=0$.

The positive sign of the exponents in these relations between probabilities and coherences is not a misprint: the coherence $\rho_{m}^{k}=\rho_{m+k, m-k}$ does decay more slowly than the density $\rho_{m}=\rho_{m, m}$. Moreover, there is no conflict with the nowadays popular phenomenon of accelerated decoherence [12,13, 15]: Quantum dissipative processes do imply much larger decay rates for coherences than for probabilities but only so with respect to certain states which are distinguished by the process itself; for the dissipative process studied here such distinguished states are, for instance, coherent angular-momentum states 14,10 but not the states $|j m\rangle$.

A simple illustration of the statement just made may be helpful, even if it amounts to sidestepping to another dissipative process for an angular momentum, the one described by the master equation $[6] \dot{\rho}=\kappa\left\{\left[J_{z}, \rho J_{z}\right]+\left[J_{z} \rho, J_{z}\right]\right\}$. In that case the eigenstates $|j m\rangle$ of $J_{z}$ are the distinguished ones as is obvious from $\dot{\rho}_{m}^{k}=-4 \kappa k^{2} \rho_{m}^{k}$ : The probabilities $\rho_{m}^{0}$ are all conserved while the coherences have decay rates growing quadratically with the skewness $\mathrm{k}$.

\section{Saddle-point evaluation of the Laplace integral}

The relation (3.6) between probabilities and coherences clearly allows us to confine the remaining investigation to the case $k=0$, i.e. to the propagator of the densities. Our goal is to do the integral in the exact formula (3.5) in the limit of large $J$. To begin with, let us rewrite that formula for $k=0$ as

$$
D_{m n}(\tau)=\frac{Q_{m n}}{2 \pi i} \int_{b-i \infty}^{b+i \infty} \mathrm{e}^{Z_{m n}(v, \tau)} d v
$$

with the exponent

$$
Z_{m n}(v, \tau)=\tau v / J-\sum_{l=m}^{n} \ln \left(v+g_{l}\right) .
$$

Suppose now that $n-m \gg 1$. Then since the number of terms in the sum $Z$ is proportional to $n-m$ its value is generally also large, which fact suggests a saddle-point approximation. The stationary points of the exponent are given by the solutions for $v$ of

$$
Z_{m n}^{\prime}=\tau / J-\sum_{l=m}^{n} \frac{1}{v+g_{l}}=0
$$

All roots of this saddle-point equation are real as is immediately seen by putting $v=x+i y$ and separating the imaginary part. We further note that to the right of the largest pole $v_{\max }=\max _{m \leq l \leq n}\left\{-g_{l}\right\}$ of the integrand in (3.5) the sum in (3.10) decreases monotonically from $+\infty$ to 0 as $v$ grows from $v_{\max }$ to infinity. Therefore we have one and only one root $v_{0}$ in that domain. Its position depends on the time $\tau$ : When $\tau$ goes to zero $v_{0}$ tends to infinity; conversely, for $\tau \rightarrow \infty$ the saddle point $v_{0}$ approaches the pole at $v_{\max }$.

The second derivative with respect to $v$ of the exponent,

$$
Z_{m n}^{\prime \prime}=\sum_{m}^{n} \frac{1}{\left[v+g_{l}\right]^{2}}
$$

is positive for real $v$ which means that the direction of steepest descent from the saddle is parallel to the imaginary axis. The saddle-point approximation for the integral (3.8) thus gives

$$
D_{m n} \approx \frac{Q_{m n}}{\sqrt{2 \pi Z_{m n}^{\prime \prime}}} \mathrm{e}^{Z_{m n}\left(v_{0}, \tau\right)}
$$

\section{Euler-Maclaurin estimates for the sums}

To render the expression (3.12) useful, we must evaluate the three sums in $Z_{m n}, Z_{m n}^{\prime}, Z_{m n}^{\prime \prime}$. The familiar EulerMaclaurin summation formula $\sum_{m}^{n} f(k) \approx \int_{m}^{n} f(x) d x+(f(m)+f(n)) / 2$ comes to mind first but is not immediately suitable for our purpose. We rather employ a modified version which involves nothing but an integral; to compensate for the absence of the extra boundary terms the integration interval is extended, 


$$
\sum_{m}^{n} f(k) \approx \int_{m-1 / 2}^{n+1 / 2} f(x) d x .
$$

The accuracy of both summation rules is the same for smooth summands $f(k)$.

In applying (3.13) to the sum in the saddle-point equation we rewrite the rate function as $g_{l}=J^{2}-(l-1 / 2)^{2}$, introduce the rescaled variables

$$
\mu=\frac{m-1}{J}, \quad \nu=\frac{n}{J}, \quad a=\frac{\sqrt{v_{0}+J^{2}}}{J}
$$

and obtain

$$
\sum_{l=m}^{n} \frac{1}{v+g_{l}} \approx \int_{m-1}^{n} \frac{d x}{v_{0}+J^{2}-x^{2}}=\frac{1}{2 J a} \ln \left[\frac{(a+\nu)(a-\mu)}{(a-\nu)(a+\mu)}\right] .
$$

The saddle-point condition (3.10) thus takes the form

$$
\tau=\frac{1}{2 a} \ln \frac{(a+\nu)(a-\mu)}{(a-\nu)(a+\mu)} .
$$

It determines $a$ as a function of $\nu, \mu$, and $\tau$. As already explained above, the single root of interest is positive and larger than the larger of $|\mu|,|\nu|$.

Similary proceeding with the sums in $Z_{m n}^{\prime \prime}$ and $Z_{m n}$ we find

$$
\begin{aligned}
J^{3} Z_{m n}^{\prime \prime} & =\left.\frac{1}{2 a^{2}}\left(\tau+\frac{\nu}{a^{2}-\nu^{2}}-\frac{\mu}{a^{2}-\mu^{2}}\right)\right|_{a=a(\mu, \nu, \tau)} \equiv \Xi(\mu, \nu, \tau), \\
Z_{m n}(v, \tau) & =J\left[\tau\left(a^{2}-1\right)-2(\nu-\mu) \ln J+2(\nu-\mu)-\sigma(a, \mu, \nu)\right]
\end{aligned}
$$

with the auxiliary function

$$
\begin{aligned}
\sigma(a, \mu, \nu) & \equiv(\nu+a) \ln (\nu+a)-(\mu+a) \ln (\mu+a) \\
& -(a-\nu) \ln (a-\nu)+(a-\mu) \ln (a-\mu) .
\end{aligned}
$$

We should comment on the slight asymmetry in the definitions of the macroscopic variables $\mu$ and $\nu$ in (3.14). The use of $(m-1) / J$ instead of $m / J$ as the macroscopic variable $\mu$ is formally related to our extension by 1 of the integration interval in the summation formula $(3.13)$ and has the benefit of preventing the small parameter $1 / J$ from appearing explicitly in the saddle-point equation (3.16).

\section{UNIFORM ASYMPTOTICS OF THE PROPAGATOR}

We came to our saddle-point approximation assuming that the number of terms in the sum $Z_{m n}$ equal to $n-m$ is large. It is not surprising therefore that the approximation (3.12) loses its accuracy when $n-m$ is of the order unity or zero; that situation prevails, e.g., for small times $\tau$; an alternative approximation is then desirable and will be constructed presently.

\section{A. Small-time approximation}

To explain the essence of the new approximation let us give a simple example. Consider the Laplace image function with two simple poles $\mathcal{V}(z)=(z-c-d)^{-1}(z-c+d)^{-1}$ and its original function $V(t)=\mathrm{e}^{c t} d^{-1} \sinh t d$. As long as $t d \ll 1$ the hyperbolic sine can be replaced by its argument such that $V(t) \approx t \mathrm{e}^{c t}$. We have thus in effect replaced the two close by poles of the Laplace image by a single second-order pole; that replacement is obviously justified for sufficiently small times.

To employ this observation for the Laplace representation of the propagator (3.5) we introduce the new integration variable $x=\tau v / J$ and obtain

$$
D_{m n}(\tau)=Q_{n m}\left(\frac{\tau}{J}\right)^{n-m} \frac{1}{2 \pi i} \int_{b-i \infty}^{b+i \infty} \frac{\mathrm{e}^{x} d x}{\prod_{l=m}^{n}\left[x+g_{l} \tau / J\right]} .
$$


The length of the interval on which the poles of the integrand now lie is proportional to $\tau$,

$$
\left|g_{m}-g_{n}\right| \frac{\tau}{J}=\frac{|m+n-1|}{J}(n-m) \tau .
$$

If that length is much smaller than unity the poles of the integrand of (4.1) are nearly degenerate, and that proximity enables us to replace the product in the denominator by the $(n-m)$-th power of the average factor $x+\bar{g} \tau / J$ with $\bar{g} \equiv g_{\frac{m+n}{2}}=J^{2}-\left(\frac{n+m-1}{2}\right)^{2}$. The integral is then easily calculated and yields the small-time asymptotics of the dissipative propagator,

$$
D_{m n}(\tau)=\frac{Q_{m n}}{(n-m) !}\left(\frac{\tau}{J}\right)^{n-m} \exp \left\{-\frac{\tau}{J}\left[J^{2}-\left(\frac{n+m-1}{2}\right)^{2}\right]\right\} .
$$

Unlike the saddle-point approximation, the foregoing expression is fully explicit. We shall keep referring to it as the small-time approximant although the underlying small parameter is the combination (4.2) of both $\tau$ and the quantum numbers $m, n$.

\section{B. Matching the two approximations}

The saddle-point and the small-time approximations for the propagator practically coincide for an intermediate range of arguments. Let us assume $l=n-m+1 \gg 1$ but on the other hand $\zeta \equiv l / J \ll 1($ say, $l \sim \sqrt{J})$. The solution $a(\tau, \mu, \nu)$ of the saddle-point equation (3.16) can then be found by expanding in powers of $\zeta$,

$$
a^{2}=\nu^{2}+\frac{\zeta}{\tau}-\zeta \nu+\mathcal{O}\left(\zeta^{2}\right) .
$$

The exponent (3.18) in the saddle-point formula then simplifies according to $\tau\left(a^{2}-1\right) \approx \zeta+\tau\left[(\nu-\zeta / 2)^{2}-1\right]$ and $\sigma(a, \mu, \nu) \approx \zeta\left(\ln \frac{\zeta}{\tau}+2\right)$ while the prefactor becomes $\Xi \approx \tau^{2} / \zeta$. Collecting these pieces in (3.12) we obtain

$$
D_{m n}=\left.\frac{Q_{m n} \sqrt{l}}{\sqrt{2 \pi}}\left(\frac{\mathrm{e}}{l}\right)^{l}\left(\frac{\tau}{J}\right)^{l-1} \mathrm{e}^{-\frac{\tau}{J}\left[J^{2}-\left(n-\frac{l}{2}\right)^{2}\right]}\right|_{l=n-m+1}
$$

This in turn is the small-time approximation (4.3) provided we there replace the factorial $(n-m) !=(l-1)$ ! à la Stirling, $(l-1) ! \approx \sqrt{\frac{2 \pi}{l}}\left(\frac{l}{\mathrm{e}}\right)^{l}$. Hence the saddle-point and small-time approximations agree for $1 \ll l \ll J$.

\section{Uniform approximation}

The two approximations under discussion can be merged into a single one which generally behaves like the saddlepoint formula (3.12) but preserves its accuracy even when $m$ is close to $n$ and/or the time $\tau$ is small. We just have to divide the saddle-point result (3.12) by the ratio of the factorial $(n-m)$ ! to its Stirling approximant. If $n-m$ is large that ratio is unity but otherwise the correction replaces the saddle-point version with the small-time propagator (4.3). We thus obtain the principal result of our paper for the density propagator in the large- $j$ limit,

$$
\begin{array}{r}
D_{m n}=\frac{Q_{m n} J^{3 / 2}}{(l-1) ! \sqrt{l \Xi}}\left(\frac{l \mathrm{e}}{J^{2}}\right)^{l} \mathrm{e}^{J\left[\tau\left(a^{2}-1\right)-\sigma(a, \mu, \nu)\right]}, \\
l=n-m+1, \quad \mu=(m-1) / J, \quad \nu=n / J, \quad a=a(\mu, \nu, \tau) .
\end{array}
$$

It is valid in a wide range of quantum numbers and propagation times and thus merits the name uniformly asymptotic propagator. The error is of order $1 / J^{2}$ except for the not very interesting late times when the bulk of the probability has settled in the lowest level; that latter restriction for $\tau$ arises due to the close encounter of saddle and pole mentioned in Sect. IIIC.

We have checked that (4.6) provides an efficient tool to numerically calculate the dissipative propagator; if $j$ is large its accuracy becomes comparable or even superior to that of the numerical integration of the master equation. The only inconvenience is the necessity to determine the saddle-point parameter $a=a(\mu, \nu, \tau)$ by solving $(3.16)$ which generally has to be done numerically. 


\section{SPECIAL CASES}

We proceed to considering situations in which the uniform approximation simplifies. The strategy invariably is to approximate factorials of large numbers à la Stirling. Some cases even allow for an analytical solution for the saddle-point parameter $a$ whereupon fully explicit formulas for the propagator arise. Some well-known results of superradiance theory are thus recovered and revealed as special cases of the uniform approximation.

\section{A. Semiclassical approximation}

The uniformly asymptotic propagator (4.6) depends on the quantum numbers $m, n, j$ in two ways. First there is the factorial dependence which reflects the discrete character of the representation. Second, there is the dependence on the arguments $\mu, \nu$ which can be regarded as the classical counterparts of $m, n$ scaled with respect to the total angular momentum; they tend to continuous variables in the classical limit.

Suppose we are not interested in effects tied up with the discreteness of quantum levels and want to obtain a smooth function of the macroscopic coordinates $\mu, \nu$ only. This is easily achieved by replacing the factorials $(n-m) !,(j \pm$ $n)$ !, $(j \pm m)$ ! by their Stirling estimates. While such a replacement would be unacceptably inaccurate if the arguments $m, n$ approached $\pm j$ ("the poles" of the Bloch sphere in classical parlance) or each other, it otherwise reliably yields

$$
\begin{aligned}
D_{m n}(\tau) & =\frac{1}{\left(1-\mu^{2}\right) \sqrt{2 \pi J \Xi}} \mathrm{e}^{J \Phi(\mu, \nu, \tau)}, \\
\Phi(\mu, \nu, \tau) & =\tau\left(a^{2}-1\right)-\sigma(a, \mu, \nu)+\sigma(1, \mu, \nu) .
\end{aligned}
$$

We here speak of the semiclassical approximation because of the implied assumption that all the quantum numbers and their relevant combinations are large. As a function of $\mu$ at fixed $\nu$ and $\tau$ the semiclassical propagator displays a single maximum located according to

$$
\frac{\partial \Phi}{\partial \mu}=\ln \frac{a^{2}-\mu^{2}}{1-\mu^{2}}=0
$$

i.e. $a=1$. The saddle-point equation (3.16) then yields the most probable value of $\mu=J_{z} / J$ at time $\tau$ related to the initial value $\nu$ through

$$
\tau=\frac{1}{2} \ln \frac{(1+\nu)(1-\mu)}{(1-\nu)(1+\mu)} .
$$

Written in terms of the polar angle of the Bloch vector $\cos \Theta=\mu, \cos \Theta_{0}=\nu$ the last equation becomes the solution of the equation of motion of the overdamped pendulum (1.1) mentioned in the Introduction. Indeed, the classical picture of the atomic dynamics in superradiance is that of the Bloch vector creeping from whatever initial orientation $\theta_{0}$ towards the equilibrium $\theta=\pi$ like an overdamped pendulum with the azimutal angle $\phi=\arctan \left(J_{x} / J_{y}\right)$ fixed. None too surprisingly, the maximum of the distribution $D_{m n}$ with respect to $m$ occurs at the point $m=m(n, \tau)$ predicted by the classical motion of the Bloch vector.

As it stands in (5.1) the semiclassical propagator correctly describes a broadening of the initially sharp distribution $D_{m n}(\tau=0)=\delta_{m n}$ to one with a width $\propto \sqrt{J}$. For many applications that width is negligible such that we may replace the propagator by

$$
\lim _{J \rightarrow \infty} J D_{m n}(\tau)=\delta(\mu-\mu(\tau, \nu))
$$

where $\mu(\tau, \nu)$ is the classical trajectory according to (5.4). For instance, expectation values like $\left\langle J_{+}^{s} J_{z}^{k} J_{-}^{s}\right\rangle$ can be calculated to leading order in $J$ with the help of the foregoing sharp version of the semiclassical propagator through the integrals

$$
\left\langle J_{+}^{s} J_{z}^{k} J_{-}^{s}\right\rangle=J^{2 s+k} \int_{-1}^{1} \delta(\mu-\mu(\tau, \nu))\left(1-\mu^{2}\right)^{s} \mu^{k} d \mu=\left(1-\mu(\tau, \nu)^{2}\right)^{s} \mu(\tau, \nu)^{k},
$$

provided, it is well to repeat, the initial point $n=J \nu$ is well removed from the most highly excited ones, $j-n \gg 1$. No quantum effects at all survive in that expression; they would only show up as small standard deviations at most of order $1 / \sqrt{J}$ if the small width of the propagator (5.1) were kept. 


\section{B. Early stage of superradiant decay of highest-energy initial states}

We now take up the previously best studied aspect of superradiance, the decay of the most highly excited atomic initial states, $j-n \ll j$. We begin by studying the early stage, i.e. small $\tau$, while the bulk of the probability still resides with highly excited states. This means that only those propagator elements are significantly different from zero for which the final quantum number $m$ is also close to $j$, or $j-m \ll j$.

We are so led to examine our uniform approximation when the macroscopic variables $\nu$ and $\mu$ are close to unity. Expanding the solution of the saddle-point equation (3.16) in powers of $1-\nu, 1-\mu$ we find the function $a(\mu, \nu, \tau)$ in terms of the nonlinearly rescaled time

$$
\xi=\mathrm{e}^{-2 \tau}
$$

as $a \approx \frac{\nu-\mu \xi}{1-\xi}$. From here it is easy to establish the ingredients of the uniform propagator (4.6),

$$
\begin{aligned}
\left(a^{2}-1\right) \tau-\sigma & \approx(1-\nu) \ln \xi+(\nu-\mu)[\ln (1-\xi)-\ln (\nu-\mu)-\ln 2 \mathrm{e}], \\
\Xi & \approx \frac{\sinh ^{2} \tau}{\nu-\mu}, \quad Q_{m n} \approx(2 J)^{n-m} \frac{(j-m) !}{(j-n) !}
\end{aligned}
$$

which bring the propagator to the limiting form

$$
D_{m n}(\tau)=\left(\begin{array}{c}
j-m \\
j-n
\end{array}\right) \xi^{j-n+1}(1-\xi)^{n-m},
$$

known as the linear approximation describing the early stages of the superradiant process [1].

\section{Bright stage of superradiant decay of highly excited initial states}

Suppose now that the initial level is close to but the final quantum number $m$ far away from $j$ such that $j-m$ is of the order of $j$. For simplicity we shall also assume that $m$ is not close to $-j$. In classical terms, we take the Bloch vector as initially pointing almost to the north pole, but we wait long enough for it to develop a substantial component transverse to the polar orientation, i.e. a strong dipole moment; by excluding the late stages of near south polar orientation we confine ourselves to the phase of brightest radiation which actually gave rise to the term "super" radiance.

Under the limitations on $m, n$ just specified the saddle-point equation (3.16) can still be solved analytically. The important fact is that the function $a$ takes on values close to unity. More accurately, it can be shown that the difference $1-a$ is of the same order of magnitude as

$$
\delta_{\nu} \equiv 1-\nu,
$$

the deviation of the initial classical coordinate from unity. It will be convenient to introduce the quantum time shift

$$
\tau^{\prime}=\tau-\tau_{\text {class }}(\mu, \nu)
$$

where $\tau_{\text {class }}(\mu, \nu)$ denotes the classical time of travel from $\nu$ to $\mu$ given by (5.4); in the situation under study it is

$$
\tau_{\text {class }} \approx \frac{1}{2} \ln \frac{2}{\delta_{\nu}}-\frac{1}{2} \ln \frac{1+\mu}{1-\mu} .
$$

We can now write $a$ as

$$
a \approx 1-\left(1-\mathrm{e}^{-2 \tau^{\prime}}\right) \delta_{\nu} .
$$

By similarly evaluating the other ingredients in the propagator (4.6) to leading order in $\delta_{\nu}$ and in addition replacing all factorials but $(j-n) !=l$ ! by their Stirling estimates we come to

$$
D_{m n}=\frac{2}{J\left(1-\mu^{2}\right)} \frac{\left(l+\frac{1}{2}\right)^{l+1}}{l !} \exp \left[-2(l+1) \tau^{\prime}-\left(l+\frac{1}{2}\right) \mathrm{e}^{-2 \tau^{\prime}}\right]_{l=j-n} .
$$


To connect with wellknown results we ban the quantum time shift $\tau^{\prime}$ by substituting (5.11), (5.12) and introduce the rescaled variables

$$
z=2 J \mathrm{e}^{-2 \tau}, \quad x=z \frac{1-\mu}{1+\mu} .
$$

The propagator thus assumes the equivalent form

$$
D_{m n}(\tau)=\left.\frac{2}{J\left(1-\mu^{2}\right)} \frac{x^{l+1} \mathrm{e}^{-x}}{l !}\right|_{l=j-n, \mu=(m-1) / J} .
$$

The special case of full initial excitation, $l=j-n=0$, yields a distribution first derived by De Giorgio and Ghielmetti [7.8. 8 .

Contact with several previous treatments of superradiance is made by considering the bright-stage propagator (5.16) for high initial excitation as a function $D(\mu, \tau ; n)$ of the final coordinate $\mu$ and the time $\tau$ and verifying it to obey the first-order partial differential equation

$$
\frac{\partial D}{\partial \tau}=\frac{\partial}{\partial \mu}\left(1-\mu^{2}\right) D
$$

Obviously, that dynamics is devoid of quantum effects: The propagator $D$ drifts along the characteristics of 5.17 , i.e. the fully classical trajectories 5.4

$$
D(\mu, \tau ; n)=\frac{1-\nu(\mu, \tau)^{2}}{1-\mu^{2}} D(\nu(\mu, \tau), 0 ; n),
$$

where $\nu(\mu, \tau)$ is the time reversed classical trajectory obtained by solving (5.4) for $\nu$. All quantum effects inherent in the superradiant pulses then originate solely from an effective initial distribution $D(\mu, 0 ; n)$ which we read from (5.16) by there setting $\tau=0$,

$$
D(\mu, 0 ; n)=\frac{2}{J\left(1-\mu^{2}\right)(j-n) !}\left(2 J \frac{1-\mu}{1+\mu}\right)^{j-n+1} \exp \left(-2 J \frac{1-\mu}{1+\mu}\right) .
$$

We should emphasize that this effective initial distribution does not coincide with the true sharp initial form of the propagator, simply because our asymptotic propagator (5.16) is not valid at small times. The essence of the earlier theories of Refs. [7 9] is thus recovered: Each run of a superradiant decay of a highly excited atomic initial state produces a macroscopic, i.e. classical radiation pulse originating from effectively random initial data, the latter reflecting quantum fluctuations.

\section{Time dependent expectation values}

We shall here establish a master formula for the set of "moments" defined as

$$
M_{k s}(\tau ; l)=\operatorname{tr}\left[\hat{\rho}(j-l ; \tau) J_{+}^{s} J_{z}^{k} J_{-}^{s}\right]
$$

with nonnegative integers $k, s, l$ and $\hat{\rho}(j-l ; \tau)$ the density operator originating from the pure initial state $|j, j-l\rangle$. In the case of $j$ much greater than 1 and $k, s, l$ much smaller than $j$ the average $M_{k s}(\tau, l)$ can be written in the form of an integral over the classical variable $\mu$ with the propagator $J D_{m, j-l}(\tau) \equiv D(\mu, \nu, \tau)$ as a weight,

$$
M_{k s}(\tau ; l)=J^{2 s+k} \int_{-1}^{1} D(\mu, \nu, \tau)\left(1-\mu^{2}\right)^{s} \mu^{k} d \mu .
$$

Upon employing the propagator (5.16) pertinent to the most highly excited initial states, changing the integration variable to $x$ [cf. (5.15] ], and once more using the rescaled time $z$ from (5.15) we recover

$$
M_{k s}(\tau ; l)=\frac{J^{2 s+k}(4 z)^{s} \mathrm{e}^{z}}{l !} \int_{0}^{\infty} \frac{x^{l+s}(z-x)^{k}}{(z+x)^{2 s+k}} \mathrm{e}^{-x} d x,
$$

an asymptotic result found by rather different methods in [9.10]. It has a scaling form inasmuch as $M_{k s}(\tau ; l) J^{2 s+k}$ depends on $J$ and $\tau$ only through the single combination $z$. 


\section{PASSAGE TIME DISTRIBUTION}

In the classical picture of superradiance the Bloch vector starts its downward motion from a certain initial angle $\Theta_{0}$ and crosses the latitude $\Theta$ at a strictly definite time $\tau_{\text {class }}(\mu, \nu)$ with $\nu=\cos \Theta_{0}, \mu=\cos \Theta$. In other words, the classical probability density of the times of crossing a given coordinate $\mu$ on the way from the initial point $\nu$ is given by the delta function $\delta\left(\tau-\tau_{\text {class }}(\mu, \nu)\right)=\delta\left(\tau^{\prime}\right)$; the quantum time shift $\tau^{\prime}$ defined in (5.11) is strictly zero in the classical limit.

Let us now introduce the quantum mechanical generalization of the classically sharp passage time distribution. According to the master equation (2.6) for the densities, the change of the probability for the system to be in level $m$ during the time interval $d \tau$ equals $\left(g_{m+1} \rho_{m+1}-g_{m} \rho_{m}\right) d \tau$. The quantity $g_{m} \rho_{m}(\tau) d \tau$ is obviously the probability for the atoms to go down from level $m$ to level $m-1$ during the time interval $[\tau, \tau+d \tau]$ and

$$
P_{m}(\tau)=g_{m} \rho_{m}(\tau)
$$

is the corresponding probability density for the time of passage through level $m$. In particular, by stipulating the atoms to have started from the pure state $|j n\rangle$ with $n>m$, we specify the passage time distribution as proportional to the propagator,

$$
P_{m}(\tau ; n)=g_{m} D_{m n}(\tau)
$$

By simply integrating $\dot{D}_{m n}(\tau)$ as given by the master equation 2.6 ) one easily shows that our passage time distribution is properly normalized to unity,

$$
\int_{0}^{\infty} P_{m}(\tau ; n) d \tau=1
$$

Our uniform approximation for the propagator allows to easily and accurately calculate the passage time distribution. In particular, if the initial state is not close to the north pole, the function $P_{m}(\tau ; n)$ is just a somewhat widened variant of the classical delta distribution, with a width inversely proportional to the square root of the second derivative $J \Phi_{\mu \mu}$ at the maximum of the exponent in the semiclassical approximation (5.1).

However, for the more interesting initial states of highest excitation, the passage time distribution has little in common with its classical analogue. As follows from (5.14) in the case of the initial state $|j, j-l\rangle$ with $l \ll j$ we rather get

$$
P_{m}(\tau ; j-l)=\frac{2}{l !}\left(l+\frac{1}{2}\right)^{l+1} \exp \left[-2(l+1) \tau^{\prime}-\left(l+\frac{1}{2}\right) \mathrm{e}^{-2 \tau^{\prime}}\right] .
$$

This density depends only on $l$ and $\tau^{\prime}$. It gives directly the time distribution of the $m \rightarrow m-1$ transition with respect to the classical time which corresponds to $\tau^{\prime}=0$.

The absence of any explicit dependence on $m$ and $j$ means that the time distributions of probability calculated for different values of these quantum numbers but the same $l=j-n$ differ only by a trivial time shift equal to the change in the classical time $\tau_{\text {class. }}$. In particular, the standard deviation of the time of crossing the $m$ th level, $\Delta \tau=\sqrt{<\tau^{2}>-<\tau>^{2}}$ with

$$
<\tau^{k}>=\int_{0}^{\infty} \tau^{k} P_{m}(\tau ; j-l) d \tau,
$$

is a function of $l$ only. The integrals (6.5) are easily calculated and give the mean passage time and the standard deviation as

$$
\begin{aligned}
<\tau> & =\tau_{\text {class }}+\frac{1}{2}\left[\mathbf{C}+\ln \left(l+\frac{1}{2}\right)-\sum_{k=1}^{l} \frac{1}{k}\right], \\
\Delta \tau & =\frac{1}{2}\left(\frac{\pi^{2}}{6}-\sum_{k=1}^{l} \frac{1}{k^{2}}\right)^{1 / 2},
\end{aligned}
$$

where $\mathbf{C}=0.5772156649 \ldots$ is Euler's constant; in the case $l=0$ the sums over $k$ are absent.

When $l$ becomes large compared with unity the distribution (6.4) becomes sharply peaked around the point $\tau^{\prime}=0$ predicted by the classical theory. However, as long as $l$ remains of order unity or even becomes zero as for complete initial excitation the passage time distribution is rather broad: The relative standard deviation $\Delta \tau /\langle\tau\rangle$ is of order $1 / \ln j$; the small initial quantum uncertainty of the polarization $\sin \theta \approx \theta \propto 1 / \sqrt{j}$ is found to be amplified to macroscopic magnitude in the passage time. 


\section{APPENDIX: UNIFORM AND SEMICLASSICAL APPROXIMATIONS FOR THE PROPAGATOR OF COHERENCES}

The uniform approximation for the dissipative propagator of the non-diagonal elements $(k \neq 0)$ is obtained via the exact relation (3.6). In the semiclassical approximation Stirling's formula is also applied in order to replace $Q_{m \pm k}, Q_{n \pm k}$ by smooth functions of macroscopic arguments. We first note the uniform approximation for the propagator of coherences

$$
\begin{aligned}
D_{m n}^{k}= & \frac{\sqrt{Q_{m-k, n-k} Q_{m+k, n+k}}}{(l-1) !}\left(\frac{\mathrm{e} l}{J^{2}}\right)^{l} \frac{J^{3 / 2}}{\sqrt{l \Xi}} \\
& \times\left.\exp \left\{J\left[\tau\left(a^{2}-1+k^{2} / J^{2}\right)-\sigma(a, \mu, \nu)\right]\right\}\right|_{l=n-m+1} .
\end{aligned}
$$

The saddle-point parameter $a$ and the functions $\sigma, \Xi$ do not depend on $k$ and are determined in exactly the same way as for the density propagator.

Finally, we note the semiclassical approximation extending (5.1) to the propagation of coherences. Since there is an additional quantum number $k$ whose range goes to infinity when $j \rightarrow \infty$, a new macroscopic variable $\eta=k / J$ has to be introduced. It is notationally convenient to write the previously incurred function $\sigma(a, \mu, \nu)$ with the help of a new auxiliary function

$$
q(x, y)=(x+y) \ln (x+y)-(x-y) \ln (x-y)
$$

as $\sigma(a, \mu, \nu)=q(a, \nu)-q(a, \mu)$. Thus equipped we can present the propagator of the elements of the density matrix with skewness $k$ as

$$
\begin{aligned}
& D_{m n}^{k}= \frac{1}{\sqrt{\left.[1-\mu-\eta)^{2}\right]\left[1-(\mu+\eta)^{2}\right]} \sqrt{2 \pi J \Xi}} \mathrm{e}^{J \Phi^{\prime}} \\
& \Phi^{\prime} \equiv \frac{1}{2}[q(1, \nu+\eta)-q(1, \mu+\eta)+q(1, \nu-\eta)-q(1, \mu-\eta)] \\
& \quad-\sigma(a, \mu, \nu)+\tau\left(a^{2}-1+\eta^{2}\right) .
\end{aligned}
$$

Acknowledgments: This work was supported by the Sonderforschungsbereich 237 "Unordnung und große Fluktuationen". P.B. is grateful to the Department of Theoretical Physics for hospitality during his stay in Essen. He also acknowledges support of RFFI under grant number N96-02-17937. D.B. would like to thank P.B. for hospitality during his stay in St.Petersburg.

[1] M.Gross and S.Haroche, Physics Reports (Review Section of Physics Letters), 93, N5, 301-396 (1982)

[2] M.G. Benedict, A.M. Ermolaev, V.A. Malyshev, I.V., Sokolov, E.D. Trifonov, Superradiance: Multiatomic Coherent Emission, Institute of Physics Publishing, Bristol and Philadelphia (1996)

[3] R.H. Dicke, Phys. Rev. 93, 99 (1954)

[4] R. Bonifacio, P.Schwendimann, and F.Haake, Phys. Rev. A4, 854 (1971) and ibid. A4, 302 (1971)

[5] G.S. Agarwal, Phys. Rev. A2,2038 (1970)

[6] F. Haake, Quantum Signatures of Chaos, Springer, Berlin (1991)

[7] V.De Giorgio and F.Ghielmetti, Phys.Rev.A4, 2415 (1971)

[8] V.De Giorgio, Optics Commun. 2, 362 (1971)

[9] F.Haake and R.Glauber, Phys. Rev. A5, 1457 (1972)

[10] R.Glauber and F.Haake, Phys. Rev. A12, 357 (1975)

[11] M. Gross, C. Fabre, P. Pillet, and S. Haroche, Phys. Rev. Lett. 36, 1035 (1976); M.Gross, P. Goy, C. Fabre, S. Haroche, and J.M. Raimond, Phys. Rev. Lett. 43, 343 (1979)

[12] A.O. Caldeira and A.J. Leggett, Phys. Rev. A31, 1059 (1989)

[13] W. H. Zurek, Physics Today 44 (10), 36 (1991)

[14] F.T. Arecchi, E. Courtens, G. Gilmore, and H. Thomas, Phys. Rev. A6, 2211 (1972)

[15] F. Haake and M. Żukowski, Phys. Rev. A47, 2506 (1993)

[16] M.Gutzwiller, Chaos in Classical and Quantum Mechanics, Springer, N.Y., (1991) 